\title{
Technical Note on Using the Movement Velocity to Estimate the Relative Load in Resistance Exercises - Response
}

Dear Editor,

Thank you for the opportunity to respond to the points raised by Naclerio \& Larumbe-Zabala regarding our recent article [5] in Sports Medicine International Open. This study provided a detailed description of the load-velocity relationship in the full back squat exercise along with the novel and very important applications that can be derived from this relationship for the practice of resistance exercise.

We are pleased that our data have provided stimulus for further inquiry. However, we would like to point out that most of the criticisms expressed by Naclerio \& Larumbe-Zabala in their Letter to the Editor are directed towards an article published in 2010 in another scientific journal [1] which analyzed the bench press exercise, and not to the present study which involved the back squat. However, since both studies are related in many ways and they form the basis of the velocity-based resistance training line of research pioneered by our group and developed over the last two decades, we are happy to address their concerns.

In their letter, the authors stated that the data analyses that we performed were not correct and could lead to an overestimation of the relative load. Had the authors taken the time to compare the estimations of relative load (percentage of one-repetition maximum, \% 1RM) from mean velocity (MV) data obtained when using the equations provided by us (Load $=7.5786$ $\mathrm{MV}^{2}-75.865 \mathrm{MV}+113.02$ ) [1] and them (Load = 107.75-62.97 MV) [3] for the bench press exercise, they could have easily verified that no such overestimation exists. Thus, for instance, when comparing the relative loads corresponding to MV values within a range of 1.15 to $0.20 \mathrm{~m} \cdot \mathrm{s}^{-1}$ ( 35-98\% 1RM), and calculating the loads for each $0.05 \mathrm{~m} \cdot \mathrm{s}^{-1}$ change in $\mathrm{MV}$, an almost negligible mean difference of $0.65 \%$ 1 RM exists when using our equation compared to theirs, with a minimum difference of $-0.22 \% 1 \mathrm{RM}$ (for $0.85 \mathrm{~m} \cdot \mathrm{s}^{-1}$ ) and a maximum difference of $2.99 \% 1 \mathrm{RM}$ (for $\left.0.20 \mathrm{~m} \cdot \mathrm{s}^{-1}\right)$. Our analyses cannot be so wrong when the correlation between the loads obtained using the aforementioned two equations is $r=0.9995$ within a velocity range of 1.15 to $0.18 \mathrm{~m} \cdot \mathrm{s}^{-1}$. Interestingly, this very analysis further seems to indicate that our quadratic equation does, in fact, better fit the obtained load-velocity data points. In our study [1], actual mean velocity attained with the $1 \mathrm{RM}$ load $\left(\mathrm{V}_{1 \mathrm{RM}}\right)$ was $0.16 \pm 0.04 \mathrm{~m} \cdot \mathrm{s}^{-1}$ whereas it was very similar, although somewhat more variable $\left(0.162 \pm 0.07 \mathrm{~m} \cdot \mathrm{s}^{-1}\right.$ for men $)$, in the Naclerio \& Larumbe-Zabala study [3]. When using their equation to estimate load from this MV value of $0.16 \mathrm{~m} \cdot \mathrm{s}^{-1}$, a load of $97.6 \%$ $1 \mathrm{RM}$ is obtained, whereas a load of $100.9 \%$ $1 \mathrm{RM}$ results when using ours. This indicates that their equation [3] to estimate relative load from MV does indeed deviate from the actual measured value (which it tends to slightly underestimate) more than our original equation [1], especially for the slowest velocities ( $\left.M V \leq 0.20-0.25 \mathrm{~m} \cdot \mathrm{s}^{-1}\right)$, i. e. those corresponding to the heaviest loads ( $\geq 95 \% 1 \mathrm{RM}$ ). Consequently, this recent equation [3] appears to offer little, if any, additional value compared to that published by us seven years ago [1].

In our original study [1], 56 out of the 120 well-trained men who made up the total sample performed the progressive loading test in the bench press exercise twice, being assessed on a second occasion following a period of 6 weeks of resistance training. The load (\% 1RM)-velocity data from these two tests from each subject were intentionally added to the total sample after verifying that the obtained load-velocity equations were almost identical when considering the data derived from the 120 subjects or the 176 tests as the data sample. In this regard, the caption of Figure 1 of that study [1] clearly indicates that 176 tests (not subjects) were included. The equation obtained when only considering the first test performed by each subject $(n=120)$ is now provided for anyone wishing to check the obtained findings:
MPV $=-0.00003233$ Load $^{2}-0.02022$
Load +1.881

$\left(R^{2}=0.980 ; S E E=0.060 m \cdot s^{-1} ; N=1045\right)$

where MPV corresponds to the mean propulsive velocity value [6] and Load is expressed as the percentage of $1 \mathrm{RM}$.

The obtained MPV values for each load between 30 and $100 \% 1$ RM obtained when using the above provided equation compared to the original equation [1] differ between 0 and $0.003 \mathrm{~m} \cdot \mathrm{s}^{-1}$ (three thousandths of a meter per second!). In addition, as it can be observed, the coefficient of determination $\left(R^{2}\right)$, an indicator of the goodness of the fit, remains at 0.98 . Thus, the inclusion of those 56 additional tests did not result in $\mathrm{R}^{2}$ being inflated as Naclerio \& Larumbe-Zabala have suggested in their letter. In this particular regard, the references $[2,7]$ provided by the authors are not pertinent to this question since we have only measured one variable and we are not using a multiple regression analysis. Further evidence for the validity of our findings comes from another published study of our research group [4] where the velocity- and power-load relationships of the bench press and prone bench pull exercises were compared in a different sample of 75 men. The obtained load-velocity equation $\left(R^{2}=0.97\right)$ was provided in Figure 2 of that study [4]. When again comparing the results of applying this equation [4] to those of the original one [1], within a 30-100\% 1RM load range, the maximum difference for any given percentage of $1 \mathrm{RM}$ is $0.01 \mathrm{~m} \cdot \mathrm{s}^{-1}$.

In our recent study analyzing the load-velocity relationship of the squat exercise [5], it is clearly explained and discussed (see Table 2 and Figure 3 of the article) that when data from the total sample $(n=80)$ is divided into subgroups of significantly different relative strength performance (1RM/ body mass) -something that had already been done in our original article [1]-, no differences were found for the velocity attained against each percentage of 1RM, 
mean test velocity or $\mathrm{V}_{1 \mathrm{RM}}$. This is a very important finding that reinforces the practical applicability of monitoring movement velocity during resistance training. The results obtained when applying any of the equations corresponding to the three sample subgroups ( $n=24,29$ and 27 subjects, respectively), as illustrated in Figure 3, are no different than those resulting using the equation for the total sample (Figure $2 b$ ). Moreover, the $R^{2}(0.96)$ and SEE $\left(0.06 \mathrm{~m} \cdot \mathrm{s}^{-1}\right)$ values are identical.

Another issue raised in this Letter to the Editor was the statement that using second-order polynomials would overestimate the data fit. As explained in the Methods section of our article [5], "relationships between load and velocity were studied by fitting second-order polynomials to data because they provided slightly better fits than linear functions". Within a given range of possible values (in this case values for relative load and movement velocity), the model that provides the best fit should be the one selected if we aim to obtain the most precise estimation of one variable from another (load from velocity or vice versa). Why then not use a second-order polynomial (a rather simple model), instead of a first-order one (linear equation), if it better fits the available data? In all our previously discussed studies $[1,4,5]$ we performed a comparison of the linear and quadratic fit models using the extra sum-of-squares $F$ test which can compare two models if one is a special case of the other (as it is the case with the linear and quadratic models). The null hypothesis was always to select the simpler model (linear equation) unless the $P$ value was less than 0.05 . These model comparisons were performed using Prism 6.07 for Windows (GraphPad Software Inc., La Jolla, CA, USA) and indicated that the preferred model was always the quadratic one $(P<0.0001)$. A visual inspection of the load-velocity scatter data plots (see Figures 2 and 3 of our study [5]) seems to reveal a better correspondence with the quadratic rather than the linear model. In any case, the differences obtained when fitting a linear or quadratic equation to the present data [5] are very subtle. In this regard, we have compared the MPV values obtained from a linear vs. a quadratic fit for a given load between 40-100\% 1RM and found that the linear equation seems to slightly overestimate the velocities (+0.03- $\left.0.05 \mathrm{~m} \cdot \mathrm{s}^{-1}\right)$ at both ends of the loading range ( $<50 \% 1 \mathrm{RM}$ and $>95 \% 1 \mathrm{RM}$ ) and slightly underestimate $\left(-0.02 \mathrm{~m} \cdot \mathrm{s}^{-1}\right)$ the velocities associated to the medium loads ( 65-80\% 1RM). As indicated in the Results section [5], it is remarkable that the individual quadratic fits for each test provided $a \mathrm{R}^{2}$ value of $0.995 \pm 0.004$ (range: $0.983-1.000$; $\mathrm{CV}=0.35 \%)$ for $\mathrm{MV}$ and $0.995 \pm 0.003$ (range: $0.986-1.000 ; C V=0.33 \%$ ) for MPV (similar results had been previously obtained for the bench press [1]). Taken together, we believe all these findings are so compelling that they leave no room for doubt.

With regards to the suggestion of Naclerio \& Larumbe-Zabala of using a longitudinal regression analysis, we must express reservations on the pertinence and usefulness of such type of data analysis for the kind of studies discussed here [1,3-5]. A longitudinal study refers to an investigation where subject outcomes and possibly treatments or exposures are collected at multiple follow-up times. It generally yields multiple or repeated measures on each subject. The fact that subjects need to perform several repetitions (typically 3 down to 1 as load increases) against different ascending loads does not, in our view, classify our study into this category. Performing a loading test in which progressively heavier loads are lifted until a maximum is attained (1RM in this case) is physiologically necessary in order to be able to obtain the best possible performance (expressed as fastest movement velocity in the present case) against each of those increasing loads.

We would like to recommend the authors of this Letter to the Editor to select mean velocity of the propulsive phase $[2,6]$, instead of mean velocity of the entire concentric phase, as the variable of choice in their future research since it has been shown to be a better indicator of an individual's neuromuscular potential, especially when lifting light and medium loads [6]. We also encourage them to employ well defined and extensively used terms such as "mean propulsive velocity" [6] instead of "mean accelerative velocity".

Finally, concerning the unsubstantiated claim questioning the applicability of the equations obtained in our studies $[1,5]$ to free weight exercises, we must disagree with the authors. It seems timely to reaffirm that it is precisely the $V_{1 R M}$ of each resistance training exercise which determines the mean velocity attained against each percentage of 1RM (see the Discussion section of our paper [5]). That being said, a proof of the validity of our equations for its use in free weight exercises is provided by the authors themselves [3] when they found a mean $V_{1 R M}$ for the free weight bench press of $0.16 \mathrm{~m} \cdot \mathrm{s}^{-1}$, a value almost identical to that previously reported by us $[1,4]$. Furthermore, in our extensive experience of using resistance exercise in many research settings and with different populations, as well as in the training of hundredths of athletes, from recreational to top-level professionals in many sports (including several Olympic gold medalists), these equations [1, 4, 5] (and other very similar ones previously developed) have proved perfectly valid for its use not only in guided Smith-type machines but also when training using free weights provided that the exercises are performed with a proper execution technique and an adequate range of motion.

We thank Naclerio \& Larumbe-Zabala for their interest in our studies, showing that the novelty and importance of our research has called upon their attention. We hope that this debate has been fruitful and will lead to new questions being asked and improved investigations being conducted in the near future.

\section{Conflict of Interest}

The authors declare that they have no conflict of interest.

\section{References}

[1] González-Badillo J], Sánchez-Medina L. Movement velocity as a measure of loading intensity in resistance training. Int J Sports Med 2010; 31: 347-352

[2] Jidovtseff B, Croisier JL, Scimar N, Demoulin C, Maquet D, Crielaard JM. The ability of isoinertial assessment to monitor specific training effects. J Sports Med Phys Fitness 2008; 48: 55-64 
[3] Naclerio F, Larumbe-Zabala E. Loading intensity prediction by velocity and the OMNI-RES 0-10 scale in bench press. J Strength Cond Res 2017; 31: 323-329

[4] Sánchez-Medina L, González-Badillo JJ, Pérez CE, Pallarés JG. Velocity- and power-load relationships of the bench pull vs. bench press exercises. Int I Sports Med 2014; 35: 209-216

[5] Sánchez-Medina L, Pallarés JG, Pérez CE, Morán-Navarro R, González-Badillo JJ. Estimation of relative load from bar velocity in the full back squat exercise. Sports Med Int Open 2017; 1: E80-E88
[6] Sánchez-Medina L, Pérez CE, González-Badillo JJ. Importance of the propulsive phase in strength assessment. Int J Sports Med 2010; 31: 123-129

\section{Authors}

Luis Sánchez-Medina', Jesús G. Pallarés², Ricardo Morán-Navarro², Carlos E. Pérez ${ }^{3}$, Juan José González-Badillo ${ }^{4}$

\section{Affiliations}

1 Studies, Research \& Sports Medicine Centre. Government of Navarre, Pamplona, Spain

2 Human Performance \& Sports Science Laboratory. University of Murcia, Spain

3 Sports Medicine Centre. University of Murcia, Spain

4 Faculty of Sport. Pablo de Olavide University, Seville, Spain

(ㄷ)(1) (웅 